\title{
An unusual Staphylococcus saccharolyticus spondylodiscitis post kyphoplasty: a case report
}

\author{
Marie-Charlotte Trojani ${ }^{*^{*}}$, Brigitte Lamy ${ }^{2,3,4}$, Raymond Ruimy ${ }^{2,3,4}$, Nicolas Amoretti ${ }^{5}$, Karine Risso ${ }^{6}$ and
} Christian Roux ${ }^{7}$

\begin{abstract}
Background: Staphylococcus saccharolyticus is a rarely encountered coagulase-negative, which grows slowly and its strictly anaerobic staphylococcus from the skin. It is usually considered a contaminant, but some rare reports have described deep-seated infections. Virulence factors remain poorly known, although, genomic analysis highlights pathogenic potential.

Case presentation: We report a case of Staphylococcus saccharolyticus spondylodiscitis that followed kyphoplasty, a procedure associated with a low rate but possible severe infectious complication (0.46\%), and have reviewed the literature. This case specifically stresses the risk of healthcare-associated S. saccharolyticus infection in high-risk patients (those with a history of alcoholism and heavy smoking).

Conclusion: S. saccharolyticus infection is difficult to diagnose due to microbiological characteristics of this bacterium; it requires timely treatment, and improved infection control procedure should be encouraged for highrisk patients.
\end{abstract}

Keywords: Spondylodiscitis, Kyphoplasty, Healthcare-associated infection, Case report, Staphylococcus saccharolyticus

\section{Background}

Staphylococcus saccharolyticus (formerly known as Peptococcus saccharolyticus) is a rarely encountered coagulase-negative staphylococcus and the only anaerobic species of the genus Staphylococcus [1]. Although it is usually considered a non-pathogenic microorganism of the human skin flora with no particular known tropism to generate specific infections, occasional reports suggest a pathogenic potential through miscellaneous rare deep-seated infections [2-5]. Little is known on its virulence factors, pathogenesis, and determinants of

\footnotetext{
* Correspondence: mctroj@hotmail.fr

'Département de Rhumatologie, Université Cote d'Azur, CHU de Nice, Nice, France

Full list of author information is available at the end of the article
}

infection. Recently, genome-sequencing analysis has shown that $S$. saccharolyticus possesses hyaluronidase activity (similar to that of Staphylococcus aureus), toxins of the phenol-soluble modulin family, and several quorum-sensing systems that may have a tissue-invasive potential [6].

Infectious complications after vertebroplasty/kyphoplasty are rare, but potentially serious life-threatening complications affecting the patient's functional prognosis can occur $(0.46 \%$ prevalence rate), which usually result from direct inoculation from skin flora such as Staphylococcus aureus, S. epidermidis, and Cutibacterium acnes $[7,8]$. . Here, we report the third case of spondylodiscitis due to S. saccharolyticus and the first to follow a surgical procedure such as kyphoplasty that specifically stresses

(c) The Author(s). 2020 Open Access This article is licensed under a Creative Commons Attribution 4.0 International License, which permits use, sharing, adaptation, distribution and reproduction in any medium or format, as long as you give appropriate credit to the original author(s) and the source, provide a link to the Creative Commons licence, and indicate if changes were made. The images or other third party material in this article are included in the article's Creative Commons licence, unless indicated otherwise in a credit line to the material. If material is not included in the article's Creative Commons licence and your intended use is not permitted by statutory regulation or exceeds the permitted use, you will need to obtain permission directly from the copyright holder. To view a copy of this licence, visit http://creativecommons.org/licenses/by/4.0/ The Creative Commons Public Domain Dedication waiver (http://creativecommons.org/publicdomain/zero/1.0/) applies to the data made available in this article, unless otherwise stated in a credit line to the data. 
the risk of healthcare-associated $S$. saccharolyticus infection $[2,3]$.

\section{Case presentation}

A 57-year-old man with a history of alcoholism and heavy smoking was admitted for disabling back pain. Four months earlier, he had sustained two vertebral fractures (T10 and T11) due to falling; these were treated by kyphoplasty under computer tomography $(\mathrm{CT})$ guidance. Because the back pain persisted 2 weeks after the procedure, he received a zygapophyseal joint steroid injection under CT guidance. Three days later, his C-reactive protein level was $12.5 \mathrm{mg} / \mathrm{l}$ and hyperleukocytosis was moderate $(13 \mathrm{G} / \mathrm{L}$ including $10 \mathrm{G} / \mathrm{L}$ neutrophils) and the patient had no fever. Magnetic resonance imaging (MRI) findings revealed infectious spondylodiscitis (Fig. 1). A Staphylococcus saccharolyticus isolate was recovered after $90 \mathrm{~h}$ of incubation from one single vial of a first series of three blood culture (BC) sets. The same microorganism was identified from two additional BC series collected 5 and 10 days later after 83 and $100 \mathrm{~h}$ of incubation, respectively. It was not possible to perform either culturing or molecular diagnostics using the tissue sample as the patient's condition did not permit disc biopsy. However, the findings from the blood culture tests indicated a definite diagnosis of spondylodiscitis, though a catheter or spinal device was not inserted in the patient. Using disk diffusion assay, the isolate was multi-drug susceptible including to penicillin and cefoxitin. The patient was treated with $2 \mathrm{~g}$ of amoxicillin three times a day for a total duration of 4 weeks after consultation with the infectious disease team. Pain and inflammatory syndrome both gradually regressed, and MRI performed after 12 month showed decrease in hyperintensity (Fig. 2b). No clear source of the bacteria was identified. The infection was presumably from skin and the bacteria was likely introduced in the surgical site during the kyphoplasty procedure. However, we could not identify any defect in the surgical skin preparation and infection control procedures as well as any particular event causing exposure during the kyphoplasty procedure.

\section{Discussion and conclusions}

S. saccharolyticus is a lesser known coagulase-negative staphylococcus [1]. Although it is usually considered a skin contaminant, it can cause endocarditis, bone infection, or pyomyositis, which may be associated with poor outcome (Table 1) [2-5, 9-13]. In addition to the tissueinvasive factors that have been unraveled, the dependence on anaerobic conditions is considered to favor the ability to invade human tissue, while biofilm production may contribute to the colonization of medical devices [6]. Further research is needed to understand S. Saccharolyticus virulence and the risk of infection better. In this regard, reports should also carefully consider patient comorbidities as host condition may contribute to the disease development. In this context, a literature review was conducted using the key words "Staphylococcus saccharolyticus" and "case" on the PubMed database. Eight articles were excluded after reading title and/or abstract because they were not applicable to the topic. A total of 8 articles were selected, which were used to compose the summary table (Table 1). Thus far, only 3 of the 9 reported cases have detailed host risk factors. To date,

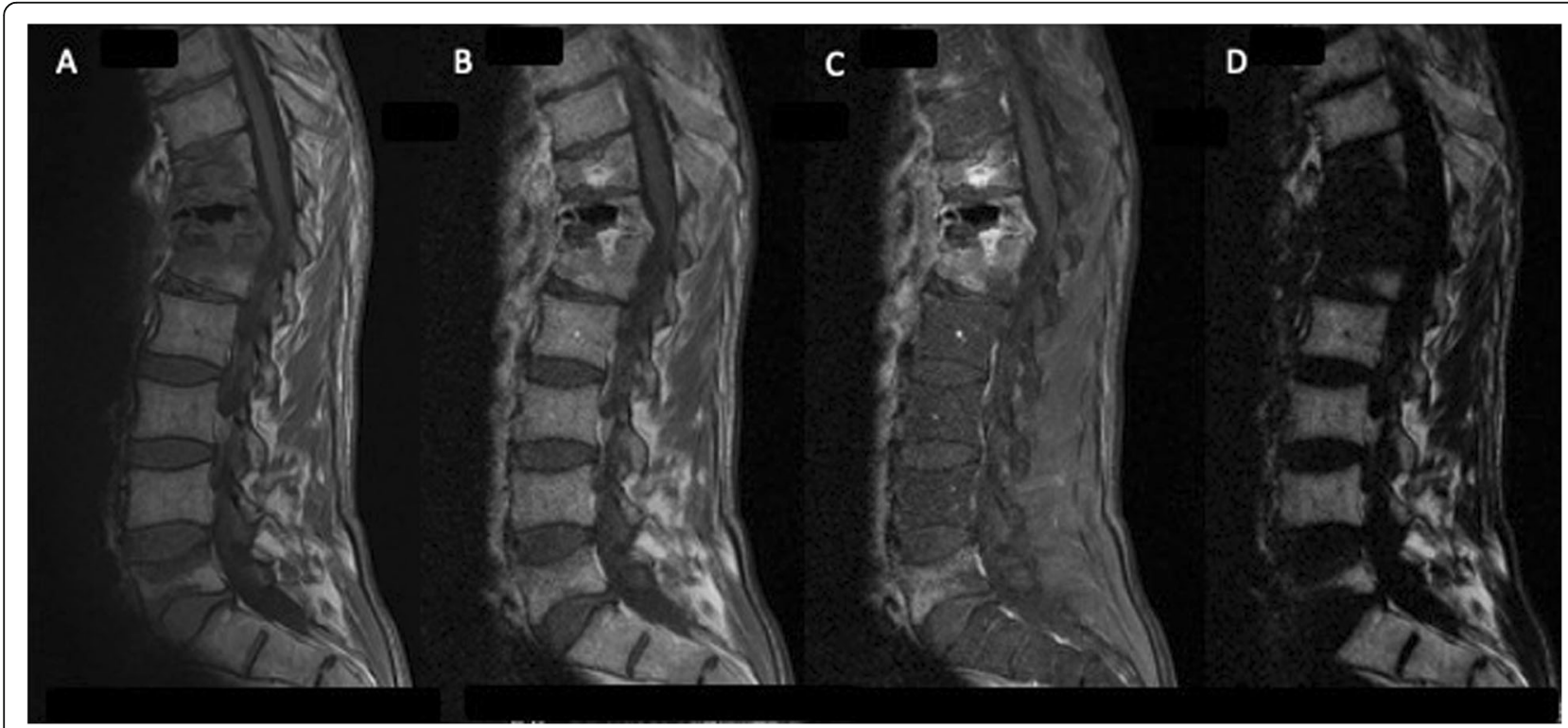

Fig. 1 Spinal MRI, sagittal section: Hyperintensity, T11-T12-L1 vertebrae, para-vertebral soft tissue, and T12-L1 disc consistent with infectious spondylodiscitis. a T1-weighted; $\mathbf{b}, \mathbf{c}, \mathbf{d}$ IDEAL sequence 


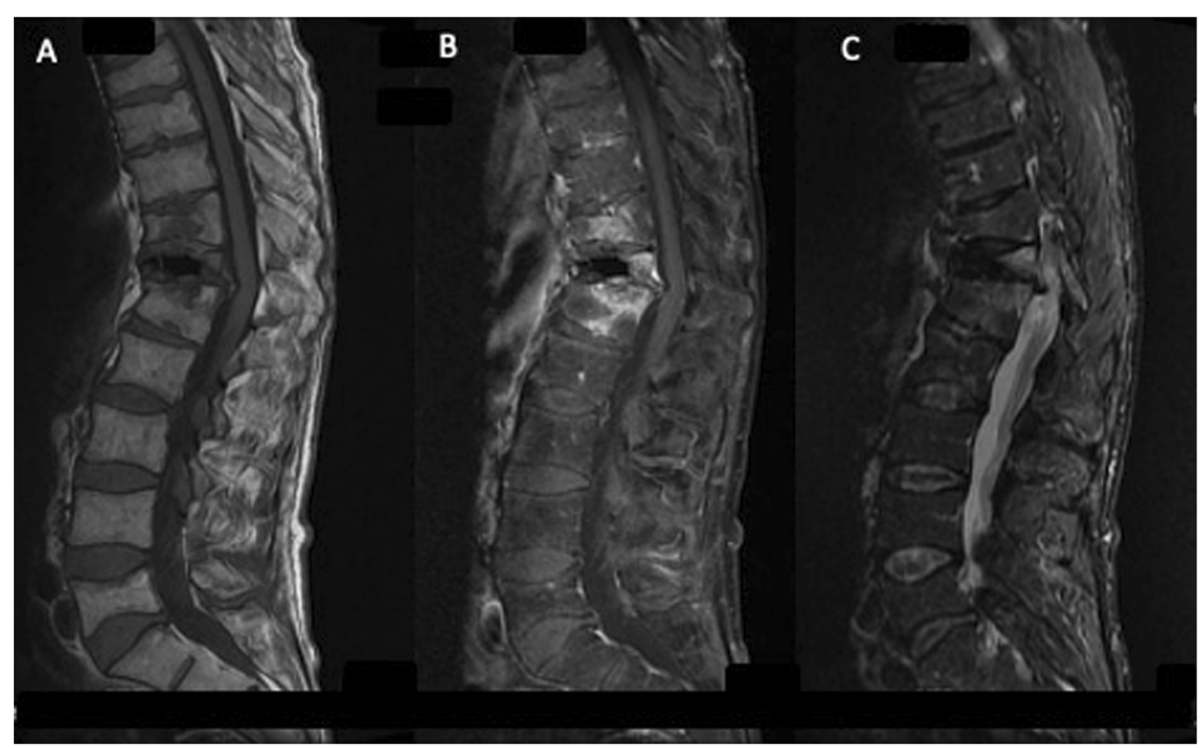

Fig. 2 Spinal MRI, sagittal section 1 year later: A decrease in signal was observed. a T1-weighted; b T1-weighted with contrast agent; c STIR sequence

only 3 cases, including our case, of spondylodiscitis have been reported, of which 1 case has been related to a surgical procedure and multiple-level diskographies [3] and 1 (current) case occurred after kyphoplasty; information on surgical procedure was not retrieved for the third case [2].

Although vertebroplasty is a minimally invasive procedure, the possibility of postoperative infection should not be ignored. It requires major salvage surgery and may lead to residual disability and even death in several cases. In addition to standard skin preparation and the administration of prophylactic antibiotics, surgeons should preoperatively consider immune status, urinary tract infection or other infection source within 6 months, and history of pulmonary tuberculosis to prevent infection post vertebroplasty [14].

It is unclear why S. saccharolyticus is specifically associated with spondylodiscitis. This either reflects a specific bacterial niche that remains to be evidenced or represents a publication bias. To date, there is no means to clarify this point. Bruggeman et al. recently reported 8 strains recovered from hip and shoulder prosthetic infections, which suggests that orthopedic sites other than the spine may be infected by S. saccharolyticus. Unfortunately, Brüggeman et al. provided no information about the clinical cases, so it is unclear if this potential contaminant was actually the causative agent of all the reported infections [6].

This case highlights several important considerations in S. saccharolyticus infection and the pitfalls associated with the diagnostic aspects. Symptoms and biological syndrome may be moderate or absent in the early stage of infection [4]. In our case, fever was absent and the inflammatory biologic syndrome was mild. Possible reasons could be the proximity of a corticosteroid injection and effective empirical treatment that was timely administered. $S$. saccharolyticus, in addition to being anaerobic, grows slowly, which may be misinterpreted as a contamination because the bacterium grows only in anaerobic bottles (not in aerobic bottles); thus, very few or only a single bottle may be positive. In addition, the long time to positivity is usually a criterion to suspect $\mathrm{BC}$ contamination (together with a single/low number of positive bottle). Thus, the characteristics of the result (long time to positivity, low number of positive bottles) could be misinterpreted for a contamination) [1, 13]. This might also lead to under diagnosis when cultures are not incubated for at least 5 days, which is a regular situation with analyses other than BC. These findings advocate for the following: i) a minimum of 5 days of anaerobic culture study when infection is strongly suspected, and no microorganism is recovered on day 3 of incubation. This implies a preferred cooperation between a rheumatologist and microbiologist to adapt and optimize the diagnostic procedures, including molecular diagnostics, when spinal infection is suspected and ii) a fine interpretation of the microbiological findings in order to prevent overlooking an infection etiology when a microorganism that is most frequently a contaminant is recovered.

The favorable evolution after appropriate antibiotics treatment is not a regular option. The rare reported infections ( 9 to our knowledge) have often been fatal ( 3 of the 7 available outcomes; Table 1). Timely treatment may be critical. Comorbidities favoring this opportunistic infection are unevenly reported: prosthetic heart valves $[9,10]$, poor oral hygiene (2), type II diabetes (5), to which we can importantly add tobacco use, alcoholism, and cachexia in this patient. 


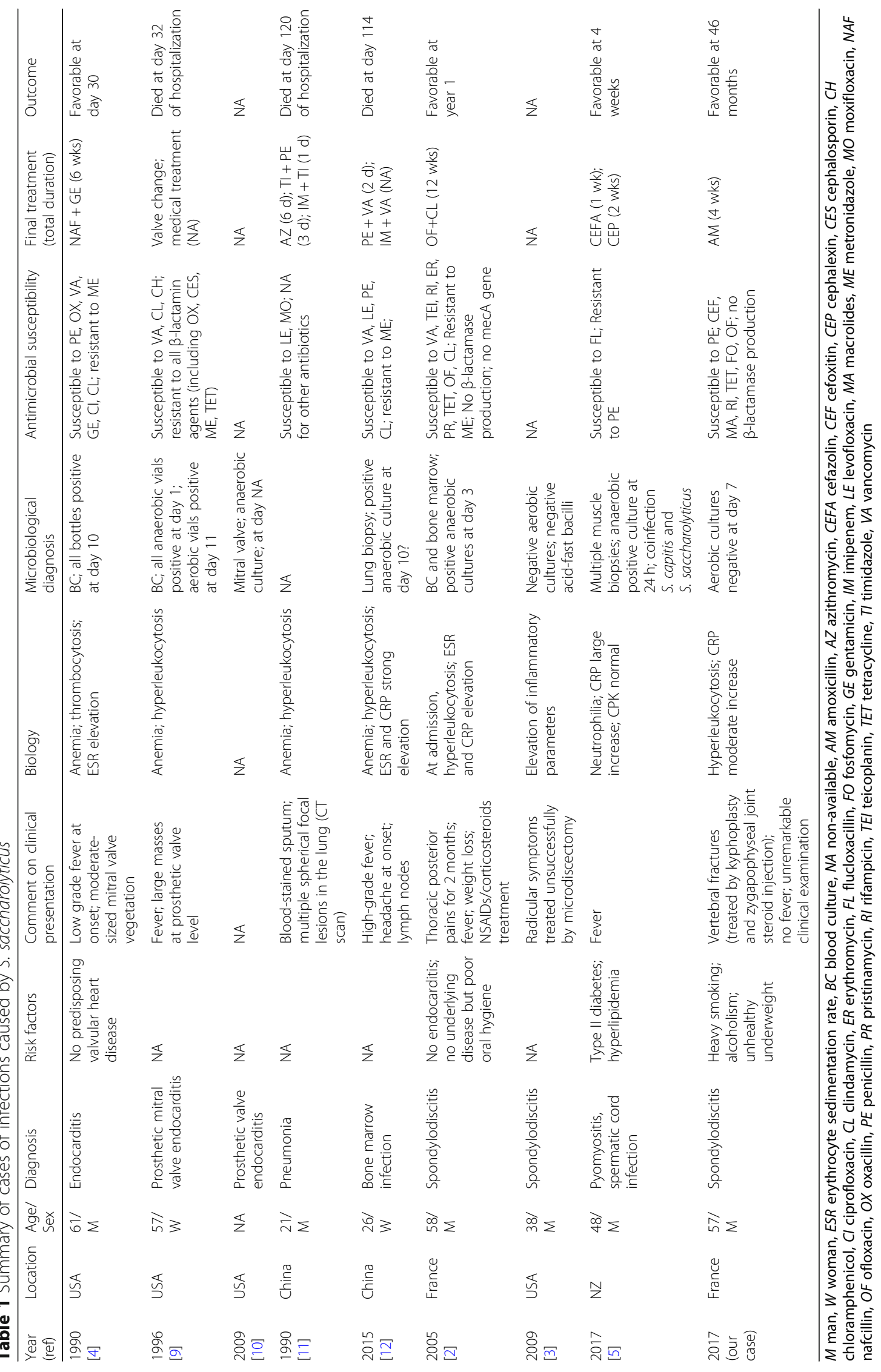


Finally yet importantly, infection control procedures designed to prevent infection following vertebroplasty procedure may require some improvements to achieve infection prevention in patients with poorer condition. This is a challenging goal because, to-date, no suggestions to help reduce the risk of infection are available in the literature when all actions taken have already complied with guidelines. Strategies for improvement may arise from further research on antibacterial advanced cement for kyphoplasty $[15,16]$ (e.g., Clarkin et al., 2011; Brauer et al., 2013), as well as from further research on improved bundle approaches. Improvements may also arise from a better understanding of the pathophysiology of surgical site infection. A step in this direction was provided by Romano-Bertrand et al. [17], who showed how disturbances of skin microbiota by antisepsis and prophylactic treatment impacted the dynamics of microbiota in deep tissues during cardiac surgery. Although this model does not exactly fit with kyphoplasty, it does clearly show that diverse bacteria may reach the surgical site during invasive procedures. Further understanding is also needed on how the patient's condition and innate immunity may impact the response towards controlling surgical site infection development during the very first steps of invasive procedures. In conclusion, the incidence of $S$. saccharolyticus spondylodiscitis is reportedly low, but clinicians must not fail the diagnosis. We advise that any $S$. saccharolyticus culture in the context of fever and/or orthopedic pain should be cautiously reviewed before being considered a contaminant. Prompt diagnosis and treatment is essential for an improved outcome of this severe infection and overall efforts should be made in infection control during vertebroplasty.

\section{Abbreviations}

CT: Computer tomography; BC: Blood culture; MRI: Magnetic resonance imaging

\section{Acknowledgements}

None.

\section{Authors' contributions}

$M C T$ was in charge of the case review and preparation of the manuscript. $\mathrm{CR}, \mathrm{BL}$, conceived the work, provided clinical expert opinion, and helped to draft the manuscript. KR, NA, and RR provided clinical expert opinion and revised the manuscript. All authors read and approved the manuscript.

\section{Funding}

None.

\section{Availability of data and materials}

Not applicable. Please contact authors for data requests.

\section{Ethics approval and consent to participate}

Written consent was obtained from the patient. No ethics committee was necessary for this case report.

\section{Consent for publication}

Written consent was obtained from the patient for publication of this case report. A copy of the written is available for review by Editor in Chief of this journal.

\section{Competing interests}

The authors declare that they have no competing interests.

\section{Author details}

'Département de Rhumatologie, Université Cote d'Azur, CHU de Nice, Nice, France. ${ }^{2}$ Laboratoire de Bactériologie, Hôpital L'archet 2, CHU de Nice, Nice, France. ${ }^{3}$ INSERM U1065, Centre Méditerranéen de Médecine Moléculaire, Equipe 6, Nice, France. ${ }^{4}$ Faculté de Médecine, Université Côte d'Azur, Nice, France. ${ }^{5}$ Département de Radiologie, Université Cote d'Azur, CHU de Nice, Nice, France. ${ }^{6}$ Service d'infectiologie, Université Nice Côte d'Azur, CHU de Nice, Nice, France. ${ }^{7}$ Département de Rhumatologie, Université Cote d'Azur, LAHMESS EA6309, CNRS, iBV UMR 7277, CHU de Nice, Nice, France.

Received: 17 March 2020 Accepted: 16 July 2020

Published online: 23 July 2020

\section{References}

1. Evans CA, Mattern KL, Hallam SL. Isolation and identification of Peptococcussaccharolyticus from human skin. J Clin Microbiol. 1978;7:261-4.

2. Godreuil S, Jean-Pierre H, Morel J, et al. Unusual case of spondylodiscitis due to Staphylococcus saccharolyticus. Joint Bone Spine. 2005;72:91-3.

3. Mikhael MM, Bach HG, Huddleston PM, Maus TP, Berbari EF. Multilevel diskitis and vertebral osteomyelitis after diskography. Orthopedics. 2009;32:60.

4. Westblom TU, Gorse GJ, Milligan TW, Schindzielorz AH. Anaerobic endocarditis caused by Staphylococcus saccharolyticus. J Clin Microbiol. 1990;28:2818-9.

5. Young N, Bhally H. Bilateral neck Pyomyositis caused by Staphylococcus capitis and Staphylococcus saccharolyticus in a diabetic adult. Case Rep Infect Dis. 2017;2017:3713212.

6. Brüggemann H, Poehlein A, Brzuszkiewicz E, Scavenius C, Enghild JJ, Al-Zeer MA, Brinkmann V, Jensen A, Söderquist B. Staphylococcus saccharolyticus Isolated From Blood Cultures and Prosthetic Joint Infections Exhibits Excessive Genome Decay. Front Microbiol. 2019;10:478.

7. Abdelrahman H, Siam AE, Shawky A, Ezzati A, Boehm H. Infection after vertebroplasty or kyphoplasty. A series of nine cases and review of literature. Spine J. 2013;13:1809-17.

8. Schofer MD, Lakemeier $S$, Peterlein $C D$, et al. Primary pyogenic spondylitis following kyphoplasty: a case report. J Med Case Rep. 2011;5:101. https://doi.org/10.1186/1752-1947-5-101.

9. Krishnan S, Haglund L, Ashfaq A, Leist P, Roat T. Prosthetic valve endocarditis due to Staphylococcus saccharolyticus. Clin Infect Dis. 1996;22: 722-3.

10. Bravo LTC, Oethinger MD. Staphylococcus saccharolyticus: A Rare but Important Cause of Anaerobic Endocarditis. Microbiology No. MB 08-8 (MB357). Am J Clin Pathol. 2009;131:286-99 (Abst n46).

11. Wu X, Yu C, Wang X. A case of Staphylococcus saccharolyticus pneumonia. Int J Infect Dis. 2009;13:e43-6.

12. Liu CJ, Sun B, Guo J, et al. A case of bone marrow infection by Staphylococcus saccharolyticus. Eur Rev Med Pharmacol Sci. 2015;19:1161-3.

13. Lanne S. Staphylococcus saccharolyticus: à propos d'un cas d'infection de dispositif cardiaque implantable, enjeux de l'identification et pathogénicité d'un germe méconnu. Sci Pharm. 2016; dumas-01323806

14. Liao JC, Lai PL, Chen LH, Niu CC. Surgical outcomes of infectious spondylitis after vertebroplasty, and comparisons between pyogenic and tuberculosis. BMC Infect Dis. 2018;18(1):555. https://doi.org/10.1186/s12879-018-3486-x.

15. Clarkin O, Wren A, Thornton R, Cooney J, Towler M. Antibacterial analysis of a zinc-based glass polyalkenoate cement. J Biomater Appl. 2011;26(3):27792. https://doi.org/10.1177/0885328210364430.

16. Brauer DS, Karpukhina N, Kedia G, et al. Bactericidal strontium-releasing injectable bone cements based on bioactive glasses. J R Soc Interface. 2013; 10(78):20120647. https://doi.org/10.1098/rsif.2012.0647.

17. Romano-Bertrand S, Frapier JM, Calvet B, et al. Dynamics of the surgical microbiota along the cardiothoracic surgery pathway. Front Microbiol. 2015; 5:787. Published 2015 Jan 13. https://doi.org/10.3389/fmicb.2014.00787.

\section{Publisher's Note}

Springer Nature remains neutral with regard to jurisdictional claims in published maps and institutional affiliations. 